\title{
Alternate IACUC Members: When Is the Regular Member 'Unavailable'?
}

A continuing problem for the Great Eastern University IACUC was to be able to have enough members present to conduct business. Conflicting committee meetings, vacations, forgetfulness, grant deadlines, etc., routinely took their toll. To try to overcome the problem, the Committee decided to have alternate IACUC members. The Institutional Official (IO), who had the authority to appoint IACUC members, readily agreed. So, when the Attending Veterinarian (AV) was away, another qualified veterinarian took her place. Likewise, there were alternates for most other IACUC members.

The system worked quite well, at least for a while. Then, two questions arose that led to discordant opinions among the Committee members. The first involved Designated Member reviews, a procedure that Great Eastern was using increasingly as the number of protocols being submitted continued to climb. The question was whether or not an alternate member could be a voting member of a Designated Member review group if the regular member was serving on a different Designated Member review group. Some members supported the arrangement, because the regular member simply was not present to review a specific protocol. They reasoned that it was no different from when the alternate substituted for the regular member when the latter was unavailable to discuss and vote on a specific protocol at a full Committee meeting. Other members strongly disagreed. They said that, because the regular member was actively engaged in reviewing an IACUC protocol, the alternate could not be a substitute because the regular member was actually available, but at the moment just happened to be occupied with another IACUC review.

The second question was related, and involved the semiannual inspections of the animal facilities, which included USDA- and NIH-covered species. The Committee debated whether or not an alternate member could do an inspection in one part of a building while the regular member was doing a semiannual inspection in another area. Again, one IACUC faction supported the arrangement, because the regular IACUC member was not available. Furthermore, they said, even in a worst-case scenario the alternates could be considered consultants, and the IACUC still would be responsible for the final evaluation and report of the facilities. Another faction disagreed. They interpreted the Animal Welfare Act (AWA) regulations as implying that at least two IACUC members should be on an inspection team, independent of any consultants to be used. Because the regular member was actually available and doing an IACUC inspection, they believed that the alternate could not be doing inspections as a substitute for the regular member, even in another area of the building. To make the situation murkier, one member asked, "What would happen if they did the inspections on different days, just to lighten the load on the regular member? Under that circumstance, could an alternate member do one of the inspections?"

If you were a member of the Great Eastern IACUC, how would you interpret the use of alternate IACUC members in the instances just described?

\section{Not Present $\neq$ Not Available}

\section{Faisal A. Guhad, BVM, MSc, PhD}

The Great Eastern IACUC's first problem involves the use of an alternate reviewer for a regular IACUC member who is actively engaged in reviewing other protocols and thus has inadequate time to review additional protocols. Members of the Committee who opposed the use of an alternate member in this situation are correct. According to the Office of Laboratory Animal Welfare (OLAW) clarification on the use of alternative members, "an IACUC member and his or her alternate may not contribute to a quorum at the same time or act in an official IACUC member capacity at the same time ${ }^{1}$ ". Furthermore, OLAW recommends against using the alternate member for quorum or voting "unless the member for whom they substitute is not available $\mathrm{e}^{\text {". }}$

The arguments of some of the Committee members who support the use of the alternate in this case are therefore false. They seem to be confusing 'not present' with 'not available. The Designated Member review procedure adopted at this University does not require voting unless a member of the IACUC requests a full Committee review. Even then, by serving on a different Designated Member review group, the regular member is engaged in an IACUC activity, which nullifies the services of the alternate member.

The second problem facing the Great Eastern IACUC involves semiannual inspections of the animal facility and whether an alternate member could inspect one part of a building while the regular member was inspecting another area. The IACUC members who supported this method of semiannual inspection will not be in compliance. Their reasoning that the regular IACUC member is not available is invalid, because he or she is actually doing the same inspection in another part of the building. Additionally, the alternate member cannot be considered a consultant for the same reason that the regular member is actively engaged in 
the facility inspection.

The Great Eastern IACUC is correct in appointing alternate members to help with their review problems even though the PHS Policy is silent on the issue. As stated in the introduction, the two related problems just discussed reflect lack of a clear definition about when the regular IACUC member is actually unavailable. Similarly, IACUC members need to know the difference between Designated Member and full Committee review procedures. However, some of the reasons given for appointing alternate IACUC members could be resolved by advance and proper scheduling of their IACUC business meetings. Forgetfulness cannot be a valid reason for appointing alternate members. Finally, perhaps the OLAW should provide clarification on alternate IACUC members by defining when a regular IACUC member is 'unavailable' to pre-empt similar misunderstandings in other IACUCs.

\section{Reference \\ 1. Shalev, M. Regulation Watch: OLAW clari- fies stance on use of alternate members on IACUCs and protocol and program reviews at collaborating institutions. Lab. Anim. (NY) 30(5), 16-17 (2001).}

Guhad is Assistant Professor of Comparative Medicine, Department of Comparative Medicine, Pennsylvania State University College of Medicine, The Milton S. Hershey Medical Center, Hershey, PA.

\section{Alternates}

'Instead of', Not 'in Addition to' Regular Members

\section{Mary Guaraldi, RLATG, CMAR}

As a member of the Great Eastern IACUC, I would have taken a conservative approach to the use of alternates to avoid the pitfalls that are often experienced when interpretations of regulations take place for the sake of convenience. Such interpretations generally result in noncompliance issues.
Although Great Eastern is in compliance with regard to the IO appointment of alternates and one-to-one designation of IACUC members with alternates, it seems that the two scenarios presented would result in noncompliance issues. In these two cases, the use of alternates would take place in addition to the regular member for protocol reviews and other IACUC business such as semiannual inspections.

In the case of the Designated Member review of protocols, a list of protocols under review (at a minimum) must be provided to each member of the Committee, and the written protocols or summary descriptions must be available to members upon request. All voting members have the opportunity to request full Committee review, and if this does not happen, then the Chair-appointed designated reviewer(s) assumes responsibility ${ }^{1}$. This makes each protocol review a single IACUC activity, so if both the member and his/her alternate were on separate Designated Member review groups, the vote would involve the inclusion of both the regular member and the alternate. The NIH Notice of Guidance from 12 February 2001 clearly states that an IACUC member and his/her alternate cannot contribute to a quorum or act in an official IACUC capacity simultaneously².

With regard to semiannual inspections, both PHS Policy and the AWA make the following (paraphrased) statement: The IACUC may determine the best means of conducting the evaluation, may invite ad hoc consultants to assist in the evaluation, and is ultimately responsible for the report and evaluation. One could argue that the IACUC should be able to conduct the evaluation of the program and facilities as they consider appropriate-even if it includes the use of alternates as ad hoc consultants in the inspection process ${ }^{3,4}$. A 1999 NIH Guidance stated that, for institutions that are covered by USDA animal welfare regulations, at least two IACUC members must participate in the evaluation ${ }^{5}$. One might try to argue that the member and the alternate could be on separate subcommittees, which would not appear to conflict with the AWA $^{4}$. I would disagree with this line of thinking, because the regulations consider semiannual inspection of facilities (and programs) to be a single IACUC activity (as was the protocol review process just described), even though the size of a program may require that the inspection be distributed over many days, or even months. For each reporting cycle, only a member or his/her alternate should become involved in the reporting process. Alternates may sit in on meetings and other activities, such as program evaluations, when the regular member is present, but they may not contribute to the quorum (or majority). A majority vote is necessary for the completion of the semiannual report ${ }^{2}$.

One solution might be for Great Eastern to add more full members to their Committee. The committee would have additional reviewers for the growing program but would require a larger number of people to constitute a quorum. The involvement of additional people can also be very helpful when the semiannual inspections are due.

\section{References \\ 1. ARENA/OLAW. Institutional Animal Care and Use Committee Guidebook 2nd edn B.1, C.1 (Office of Laboratory Animal Welfare, Bethesda, MD, 2002). \\ 2. National Institutes of Health. Office of Extramural Research Guidance Regarding Administrative IACUC Issues and Efforts to Reduce Regulatory Burden. Notice: OD-01- 017 (12 February 2001). \\ 3. Public Health Service. Public Health Service Policy on Humane Care and Use of Laboratory Animals IV.B (US Department of Health and Human Services, Washington, DC, 1986). \\ 4. 9 CFR, Subchapter A-Animal Welfare, Part 2. \\ 5. National Institutes of Health. Office of Extramural Research Guidance Regarding Reduction of Regulatory Burden in Laboratory Animal Welfare. Notice: OD-00- 007 (21 December 1999).}

Guaraldi is an IACUC member and Associate Scientist at Bristol-Myers Squibb Medical Imaging in North Billerica, MA, and a graduate student at the University of Massachusetts, Lowell, MA. 


\section{Alternate Doesn't Mean Extra

\author{
Jem Scott-Emuakpor, DVM and \\ Sandell L. Smith, JD
}

The purpose of alternate IACUC members should be to serve when regular members are unavailable and not simply to increase the IACUC membership when required to carry out multiple tasks. If Great Eastern needs to establish multiple Designated Member review groups or inspect multiple areas of the facility at the same time, then it would seem that either the scheduling process is not functioning adequately or the University should increase the number of regular IACUC members. Being from a small commercial laboratory with seven IACUC members, I can appreciate the inconvenience that can arise when there are not enough members present to constitute a quorum and carry out official IACUC duties. However, it is very difficult for me to understand why an institution, such as the infamous Great Eastern, would have so few regular (with corresponding alternate) IACUC members that the regular member and his/her alternate member are needed to conduct IACUC duties at the same time.

The IACUC Guidebook states: "An IACUC member and his/her alternate may not count toward a quorum at the same time or act in an official IACUC member capacity at the same time ${ }^{1}$." It seems clear that Great Eastern's regular member and alternate member will be acting "in an official IACUC member capacity at the same time," whether they are acting as voting members of separate Designated Member review groups or doing IACUC inspections in different areas of the building. Even if the IACUC inspections were done on two separate days, I believe they would still be considered as separate parts to the same official IACUC inspection; therefore, using the regular member on one day and the alternate on another would be tantamount to using them at the same time.

The alternate member should participate only in a situation in which the regu-

\section{A Word from USDA}

In response to the questions posed in this scenario, the United States Department of Agriculture, Animal and Plant Health Inspection Service, Animal Care (USDA-APHIS-AC) offers the following clarification and guidance:

Congress enacted the Institutional Animal Care and Use Committee (IACUC) to assist the research facility in maintaining compliance with the Animal Welfare Act (AWA) and regulations. It is intended to function as a measure to help meet the public concern for laboratory animal care and treatment. The AWA places a limit on the amount of representation on the IACUC from any one administrative unit ${ }^{1}$. If a research facility were to allow alternates to concurrently function with regular IACUC members, it could defeat the purpose of this control.

USDA concurs with the Office of Laboratory Animal Welfare (OLAW) guidance on the use of alternate IACUC members'. A regular member of the IACUC is considered 'unavailable' if they are entirely unable to perform their required duty (e.g., review proposed animal activities, vote at a convened meeting, or inspect the research facility's animal facilities). The AWA provides the minimum number of members needed for a properly constituted IACUC. Within the limit mentioned above, a research facility may appoint additional regular members to in order to fulfill all of the IACUC's responsibilities.

\section{References}

1. Animal Welfare Act Section 13(b)(1)(C).

2. National Institutes of Health. Office of Extramural Research guidance regarding administrative IACUC issues and efforts to reduce regulatory burden. NOTICE: NOT-OD-01-017 (12 February 2001).

Chester A. Gipson, DVM

Deputy Administrator

USDA-APHIS-Animal Care

lar member is absolutely unavailable. The regular member cannot be considered as 'unavailable' when he or she is carrying out official IACUC duties. As a side note, perhaps Great Eastern should consider hiring an IACUC Coordinator to help plan and schedule official IACUC duties more efficiently.

\section{Reference}

1. ARENA/OLAW. Institutional Animal Care and Use Committee Guidebook $2^{\text {nd }}$ edn A.2 (Office of Laboratory Animal Welfare, Bethesda, MD, 2002).

Scott-Emuakpor is Facility Veterinarian/Study Director and Smith is IACUC Chair Emeritus, Integrated Laboratory Systems, Inc., Research Triangle Park, NC. 